\title{
Handover reduction using optical matrix switch for indoor LTE cell deployment
}

\author{
Withawat Tangtrongpairoj ${ }^{\mathrm{a})}$, Takeshi Higashino, \\ and Minoru Okada
}

Graduate School of Information Science, Nara Institute of Science and Technology, 8916-5 Takayama, Ikoma, Nara 630-0101, Japan

a)withawat.tangtrongpairoj.wj4@is.naist.jp

Abstract: The Common Public Radio Interface (CPRI) and 10 Gbps Ethernet are the promising solution for connecting the baseband unit (BBU) and the remote antenna unit (RAU) of the Long-Term Evolution (LTE) system. It is also capable of deploying the radio cell at the indoor location. Since the CPRI link is based on the point-to-point link, the handover process occurs whenever the mobile user moves across the cell. Handover is more increased than outdoor location due to the limitation of transmitting power of the indoor equipment. In this paper, in order to reduce the handover, the Radio over Fiber (RoF) link is introduced instead of the point-to-point link. And the optical matrix switch controls to combine multiple cells into one cell. Simulation results show that the proposal reduces the number of handover by $38.27 \%$ compared to point-to-point based network.

Keywords: Radio over Fiber, handover reduction, optical matrix switch, LTE

Classification: Wireless Communication Technologies

\section{References}

[1] M. Paolini, "Beyond data caps. an analysis of the uneven growth in data traffic," Senza Fili Consulting, 2011.

[2] X. Wang, S. Thota, M. Tornatore, S.-S. Lee, H. H. Lee, S. Park, and B. Mukherjee, "Handover reduction via mobility-prediction-based vpon formation in optical-access-enabled cloud-ran," Optical Fiber Communications Conference and Exhibition (OFC), 2015, pp. 1-3, March 2015.

[3] M. Qian, W. Hardjawana, J. Shi, and B. Vucetic, "Baseband processing units virtualization for cloud radio access networks," IEEE Wireless Commun. Lett., vol. 4, no. 2, pp. 189-192, April 2015. DOI:10.1109/LWC.2015.2393355

[4] K. Boulos, M. E. Helou, and S. Lahoud, "Rrh clustering in cloud radio access networks," 2015 International Conference on Applied Research in Computer Science and Engineering (ICAR), pp. 1-6, Oct. 2015. DOI:10.1109/ARCSE. 2015.7338135

[5] 3GPP, "Radio interface protocol architecture (3gpp ts 25.301 v6.4.0 (200509))," http://www.qtc.jp/3GPP/Specs/25301-640.pdf, accessed May 10, 2016. 
[7] 3GPP, "General packet radio service (gprs) enhancements for evolved universal terrestrial radio access network (e-utran) access (3gpp ts 23.401 v10.4.0 (201106))," http://www.qtc.jp/3GPP/Specs/25301-640.pdf, accessed May. 10, 2016.

[8] D. Naboulsi, Analysis and exploitation of mobile traffic datasets, Ph.D. thesis, Networking and Internet Architecture [cs.NI]. INSA Lyon, 2015. English. (tel-01235304v2).

[9] M. Lacage, "Friis propagation loss model," https://www.nsnam.org/doxygen/ friis-spectrum-propagation-loss_8cc_source.html, accessed Apr. 19, 2016.

[10] M. Lacage, "Constant position mobility model," https://www.nsnam.org/ doxygen/constant-position-mobility-model_8cc_source.html, accessed Apr. 19, 2016.

[11] M. Lacage, "Random walk2d mobility model," http://www.nsnam.org/ doxygen/random-walk-2d-mobility-model_8h.html, accessed Jan. 4, 2016.

[12] B. Herman, "A3 rsrp handover algorithm," https://www.nsnam.org/doxygen/ a3-rsrp-handover-algorithm_8cc_source.html, accessed Mar. 20, 2016.

[13] L. N. Tran, E. K. Hong, and H. Liu, "A frequency domain equalization algorithm for fast time-varying fading channels," J. Commun. Networks, vol. 11, no. 5, pp. 474-480, Oct. 2009. DOI:10.1109/JCN.2009.6388391

\section{Introduction}

According to [1], 80\% of wireless data traffic originates from indoor locations. Mobile operators deploy the indoor equipment in order to build the radio access coverage. Mobile backhaul link for constructing small cell of the Long-Term Evolution (LTE) is composed of the baseband unit (BBU) and remote radio head (RRH), and these are connected by the optical link interfaces such as the Common Public Radio Interface (CPRI) or $10 \mathrm{Gbps}$ Ethernet. These optical links can contribute to deploy LTE cell at the indoor location.

A problem in the deploying cell at the indoor location is that the cell size must be smaller than outdoor location, because of the limited transmitting power of the indoor equipment. Since the CPRI and Ethernet link is based on the point-to-point link, it is anticipated that the handover process between cells often occurs, that is, the processing data stream in a BBU is required to switch to the other one whenever a mobile user moves across beyond the currently connected cell. In order to reduce the handover, the [2] configures the mobility-prediction, which the positioning technique is additionally required for tracking the current position of the mobile user. This paper proposes the other approach using the analog Radio over Fiber (RoF) transmission and the optical matrix switch. Fig. 1 shows the proposed configuration. Assuming that the LTE radio frequency (RF) transceivers are located in centralized control station (CCS), and the RF LTE signal is obtained from RF interface $(\mathrm{I} / \mathrm{F})$. The RF signal is transmitted between LTE transceiver and remote antenna unit (RAU) via the optical fiber link. At the RAU, the RF to optical (R/O) and the optical to $\mathrm{RF}(\mathrm{O} / \mathrm{R})$ interfaces convert between the optical to the $\mathrm{RF}$ domain. This paper assumes the intensity modulation-direct detection (IM/DD) as an optical I/F. Since the waveform format is analog, one optical stream can be easily distributed to multiple remote cells by dividing it passively. Therefore, RF resources of the bandwidth are shared among multiple cells using the optical matrix 


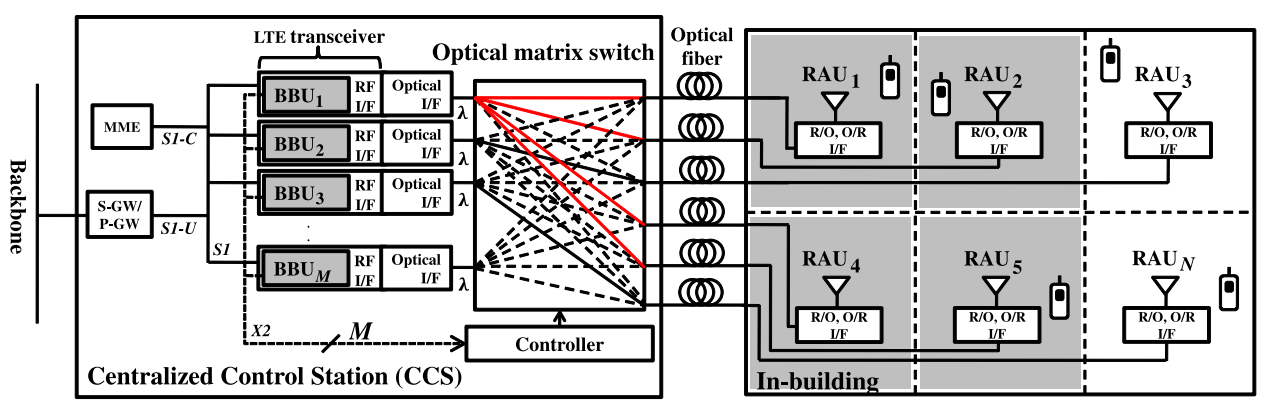

Fig. 1. Proposed RoF network with optical matrix switch

switch. The role of controller unit is to collect the resource usage of each BBU, then decides combination in the switch. The details of an algorithm are shown in Section 3.

In the proposal, a cell where a large number of mobile users exist is combined with several neighboring cells. Therefore, the handover is no longer required at combined cell even though the mobile user moves out of the cell. The primary goal of the proposal is to reduce the number of handover compared to the point-to-point based network. As the secondary advantage of the radio resource sharing among cells, the required number of LTE transceiver equipment in the CCS can be reduced. In the previous papers [3, 4], the resource management has been modeled as an optimization problem that aims to minimize the number of BBUs, which is called as the heuristic bin-packing problem. However, the goal of algorithm limited to minimize the number of BBUs, while the number of handover is still high. In proposal, the neighboring cells are combined to one cell. Hence, the resource sharing is able to reduce the BBU equipment accompany with the decreasing of the handover process. The number of BBUs, $M$, could be less than the number of RAUs, $N$.

\section{Handover procedure}

Each BBU connects with the controller via $X 2$ interface as shown in Fig. 1. The network components in the CCS are connected by the $S 1$ interface, including the mobility management entity (MME), the serving gateway (S-GW), the packet data unit gateway (P-GW) and the BBUs. The $S 1-C$ and $S 1-U$ are the interface for control plane (C-plane) and user plane (U-plane), respectively [5]. These components and connections except controller are identical to the regular LTE system. In the regular LTE for configuring small cells, the C-plane and U-plane run over the macro cell and small cell fronthaul network separately. The MME manages the handover process. In our proposal, the MME operates handover process in the CCS through the $X 2$ and $S 1-C$ interfaces. And the fronthaul elements including RAUs and fiber optics simply transfer the radio signal [6]. Therefore, the conventional handover process [7] can be introduced to our proposal without any modification in the protocol of $X 2$ and $S 1-C$ interfaces.

In the proposal, the physical cell identity (PCI) is employed to identify the BBU like regular LTE system. Multiple RAUs share one PCI when multiple cells configure to the combined one. For example, in Fig. 1, radio signal transmitted 
from RAU of $1^{s t}, 2^{\text {nd }}, 4^{\text {th }}$ and $5^{\text {th }}$ have the identical PCI when these RAUs share resource of $\mathrm{BBU}_{1}$. The handover process is activated when the mobile terminal receives LTE signal with more than one PCI. At the beginning of the process, the source $\mathrm{BBU}$ sends the request to alternative $\mathrm{BBU}$, and alternative $\mathrm{BBU}$ replies acknowledgment through the $X 2$ interface. Then, the alternative BBU sends the request to the MME via $S 1-C$ interface in order to create a new session. Details of the procedure are shown in [7]. After the mobile terminal connects to the BBU, the mobile terminal will send the user data to the S-GW and P-GW through S1-U interface.

In this proposal, the handover procedure can be categorized into two cases [8]. One occurs when the user moves across cells where the RAUs connect with different BBUs. The other occurs when the optical matrix switch changes the connection combination even through the users do not move across the cell. Since the controller requires the information on user density and estimated offered load, the proposal is activated. This paper evaluates the total number of handover for above two cases.

\section{Proposed algorithm for controlling optical matrix switch}

Fig. 2a shows the flowchart. At first, the optical matrix switch configures point-topoint link connection between BBU and RAU. We assumed that the controller has adjacency relationship for all RAUs. Let $G=(V, E)$ be a graph with $N$ vertices; each vertex represents RAU in the system. The neighborhood of vertex in graph $G$ is connected by the edge, and the weight of all edges is the same. Two functions are defined as follows, nou[ $n]$ gets the number of users connected to vertex $n$ and eol $[n]$ gets estimated offered load on vertex $n, n=1, \ldots, N$ and $|V|=N$. The controller can carry out nou $[n]$ and eol $[n]$ through the $X 2$ interface. Set $B_{m}$ is the combination set of RAU that will connect to one BBU of $m-t h, m=1, \ldots, M$. And set $R$ is $M$ set of $B$, then $R=\left\{B_{1}, B_{2}, \ldots, B_{M}\right\}$, for example, $B_{1}$ means the set of RAUs that connected to BBU of 1-st.

Fig. $2 \mathrm{~b}$ shows pseudocode. The proposal is based on the Breadth-first search. The parameters are graph $G$ and source, source is a vertex $n$ with maximum nou[ $n]$. In line $2^{\text {nd }}-14^{\text {th }}$, the source vertex and its neighbor vertices are grouped in set $B$. Then, the elements in $B$ will be assigned to one BBU. Above procedure repeats until the BBU is full usage. Then, the algorithm adds the set $B$ to $R$ as shown in line $21^{\text {th }}$. In the last process, the algorithm returns set $R$.

After updating connection combination of optical matrix switch, resource usage is periodically monitored. Another BBU is activated when the estimated usage of BBU exceeds capacity, then switch the data stream to the BBU. In contrast, BBU with small usage is deactivated after switching its data stream to another BBU. Hence above monitoring process reduces the required number of LTE transceiver in the CCS. 


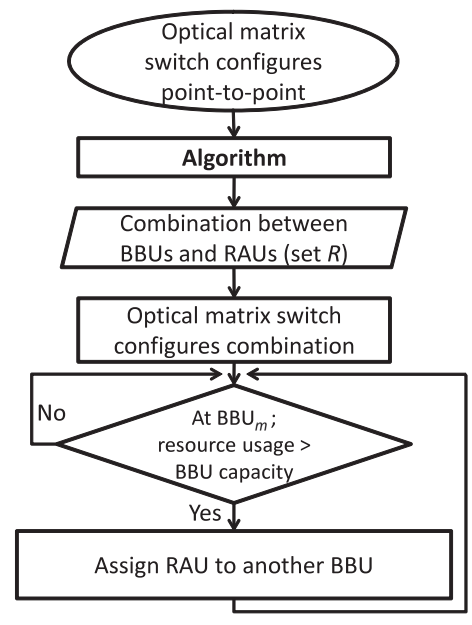

(a) Flowchart

1: function Combination $(G$, source)

2: $\quad$ create empty queue $Q$ and empty set $B$

3: $\quad t_{\text {sum }}=0$

4: $\quad t_{\text {sum }}=t_{\text {sum }}+$ eol[source]

5: $\quad$ add source to $B$

6: $\quad$ mark source in $V$

7: $\quad Q$.enqueue(source)

8: $\quad$ while $Q$ is not empty do

9: $\quad u=Q$.dequeue()

10:

for each unmarked vertex $v$ that is adjacent to $u$ do $Q$.enqueue $(v)$ if $t_{\text {sum }}+\operatorname{eol}[v] \leq \mathrm{BBU}$ capacity then

$t_{\text {sum }}=t_{\text {sum }}+\operatorname{eol}[v]$

add $v$ to $B$

mark $v$ in $V$

if all vertices in $G$ is marked then

$$
\begin{aligned}
& R \leftarrow R \cup B \\
& \text { return } R
\end{aligned}
$$

\section{end if}

else

$$
R \leftarrow R \cup B
$$

$w \leftarrow$ unmarked vertex $n$ with maximum nou $[n]$

$$
\operatorname{Combination}(G, w)
$$

end if

\section{end for}

end while

end function

\section{(b) Algorithm}

Fig. 2. Flowchart and algorithm 


\section{Network simulation}

The network simulation version 3 (ns-3) is introduced for performance evaluation. The assumption is as follows. The number of RAUs was 18. The RAU was attached on the hall roof, and its transmission power was $+10 \mathrm{dBm}$ with the center frequency of $1.93 \mathrm{GHz}$. The RAUs were deployed in a grid formation with 3 rows and 6 columns, and were spaced by 30 meters.

The free-space model [9] was employed as path loss between RAU and mobiles. As an initial step, all of the mobiles were uniform randomly placed on the field before running the simulation. Two models for simulating behavior of mobile user were employed: one-way constant walk mobility model [10] and random walk two-dimensional mobility model [11], with the speed of $2 \mathrm{~m} / \mathrm{s}$. The A3Rsrp [12] was employed as a handover scheme, which is a typical method in LTE system. The hysteresis of $3.0 \mathrm{~dB}$ and time to trigger of $256 \mathrm{~ms}$ were set as parameters. Each mobile user randomly requested the data rate of $4 / 12$ / $20 / 40 \mathrm{Mbps}$ with its distribution ratio of $0.5 / 0.3 / 0.1 / 0.1$, respectively. The capacity of a BBU was $200 \mathrm{Mbps}$. One simulation lasted 60 seconds on the simulation time.

It is anticipated that strong fading in radio channels must be generated at the overlapped area when the multiple cells are combined into one cell by the optical matrix switch. This kind of artificial fading occurs in both uplink and downlink radio channel. In our assumption, fading can be compensated by the channel equalization technique equipped in LTE system such as frequency domain equalization (FDE) [13]. The performance evaluation assumes that fading has negligible impact on the process of handover and also the number of handover.

Fig. 3a and Fig. 3b show the performance comparison when the mobility model is one-way constant. Our proposal can reduce the number of handover by $38.27 \%$ compared with the point-to-point based network. Besides, the number of BBUs is a bit higher than the bin-packing algorithm. In this figure, all of the RAUs are connected to one BBU in both of proposal and bin-packing in the case of the average number of user is 1 . The handover process driven by user mobility were not occurred. The number of handover of 0.28 occurred by only for control the switch configuration. Fig. $3 \mathrm{c}$ and Fig. $3 \mathrm{~d}$ show the performance when the mobility model is random walking. The proposal can reduce the number of handover by $36.12 \%$ and $23.25 \%$ compared to point-to-point based network and bin-packing algorithm, respectively. It is found that there is a trade-off between the number of BBUs and the number of handover. 


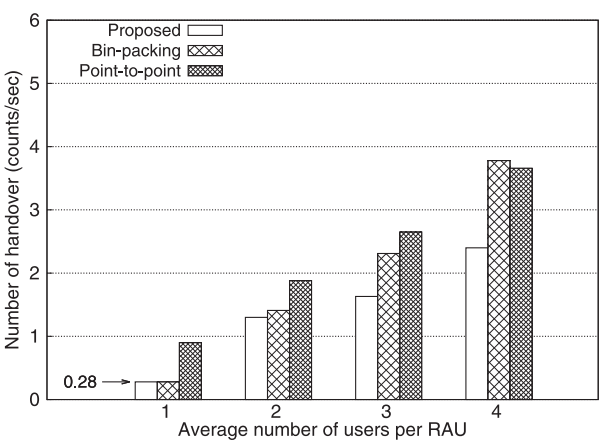

(a) Number of handover (One-way constant walk)

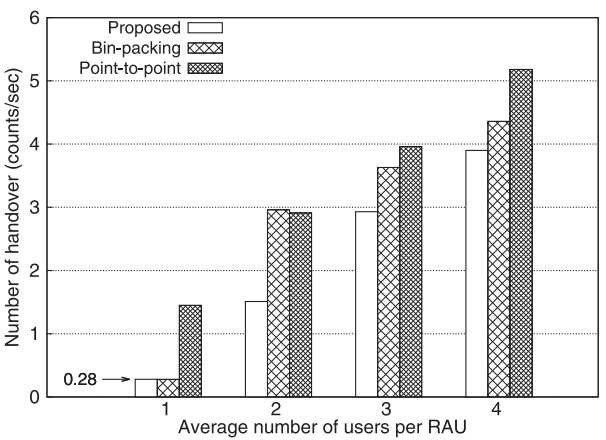

(c) Number of handover (Random walk)

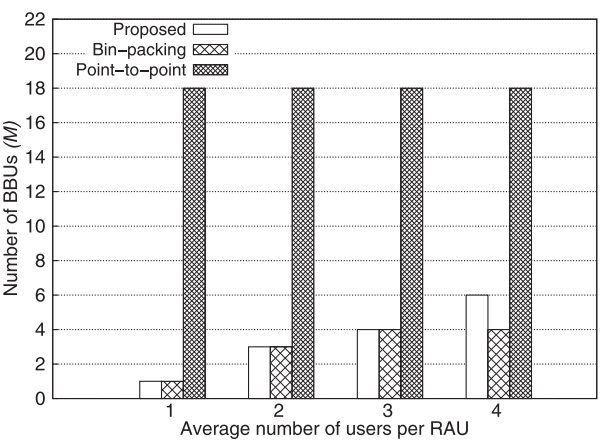

(b) Number of BBUs (One-way constant walk)

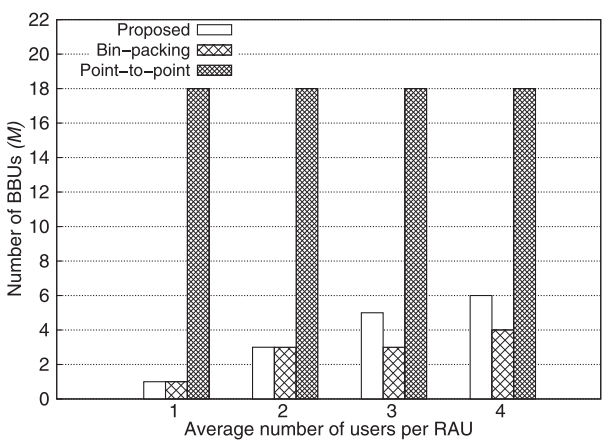

(d) Number of BBUs (Random walk)

Fig. 3. Simulation results for the number of handover (counts/sec) (a), (c) and number of BBUs (b), (d)

\section{Conclusion}

In this paper, we proposed a new mobile fronthaul configuration using the optical matrix switch and RoF link for LTE cell deployment at the indoor location. The proposal combines several cells into one cell in order to reduce the number of handover accompany with the number of BBUs equipment even though the handover which is not driven by user mobility presents. Our proposal can reduce the number of handover by $38.27 \%$ and $36.12 \%$ compared with the point-to-point based network when the mobility models are one-way constant and random walking, respectively. 